\title{
SHARP FRACTIONAL HARDY INEQUALITIES IN HALF-SPACES
}

\author{
RUPERT L. FRANK AND ROBERT SEIRINGER \\ Dedicated to V. G. Maz'ya
}

\begin{abstract}
We determine the sharp constant in the Hardy inequality for fractional Sobolev spaces on half-spaces. Our proof relies on a non-linear and non-local version of the ground state representation.
\end{abstract}

\section{INTRODUCTION AND MAIN RESULTS}

This short note is motivated by the paper BD concerning Hardy inequalities in the half-space $\mathbb{R}_{+}^{N}:=\left\{\left(x^{\prime}, x_{N}\right): x^{\prime} \in \mathbb{R}^{N-1}, x_{N}>0\right\}$. The fractional Hardy inequality states that for $0<s<1$ and $1 \leq p<\infty$ with $p s \neq 1$ there is a positive constant $\mathcal{D}_{N, p, s}$ such that

$$
\iint_{\mathbb{R}_{+}^{N} \times \mathbb{R}_{+}^{N}} \frac{|u(x)-u(y)|^{p}}{|x-y|^{N+p s}} d x d y \geq \mathcal{D}_{N, p, s} \int_{\mathbb{R}_{+}^{N}} \frac{|u(x)|^{p}}{x_{N}^{p s}} d x
$$

for all $u \in C_{0}^{\infty}\left(\overline{\mathbb{R}_{+}^{N}}\right)$ if $p s<1$ and for all $u \in C_{0}^{\infty}\left(\mathbb{R}_{+}^{N}\right)$ if $p s>1$. In [BD the sharp (that is, the largest possible) value of the constant $\mathcal{D}_{N, 2, s}$ for $p=2$ is calculated. Our goal in this paper is to determine the sharp constant $\mathcal{D}_{N, p, s}$ for arbitrary $p$.

Indeed, we shall see that the sharp inequality (1.1) follows by a minor modification of the approach introduced in [FS]. In that paper we calculated the sharp constant $\mathcal{C}_{N, p, s}$ in the inequality

$$
\iint_{\mathbb{R}^{N} \times \mathbb{R}^{N}} \frac{|u(x)-u(y)|^{p}}{|x-y|^{N+p s}} d x d y \geq \mathcal{C}_{N, p, s} \int_{\mathbb{R}^{N}} \frac{|u(x)|^{p}}{|x|^{p s}} d x
$$

for all $u \in C_{0}^{\infty}\left(\mathbb{R}^{N}\right)$ if $1 \leq p<N / s$ and for all $u \in C_{0}^{\infty}\left(\mathbb{R}^{N} \backslash\{0\}\right)$ if $p>N / s$. A (non-sharp) version of (1.2) was used by Maz'ya and Shaposhnikova [MS] in order to simplify and extend considerably a result of Bourgain, Brezis and Mironescu [BBM] on the norm of the embedding $\dot{W}_{p}^{s}\left(\mathbb{R}^{N}\right) \subset L_{N p /(N-p s)}\left(\mathbb{R}^{N}\right)$. Our proof of (1.2) relied on a ground state substitution, that is, on writing $u(x)=\omega(x) v(x)$ where $\omega(x)=$ $|x|^{-(N-p s) / p}$ is a solution of the Euler-Lagrange equation corresponding to (1.2). In this note we shall prove (1.1) using that $\omega(x)=x_{N}^{-(1-p s) / p}$ satisfies the Euler-Lagrange equation corresponding to (1.1).

We refer to $[\mathrm{BD}, \mathrm{D},[\mathrm{KMP}]$ and the references therein for motivations and applications of fractional Hardy inequalities.

Support through DFG grant FR 2664/1-1 (R.F.) and U.S. NSF grants PHY-0652854 (R.F.) and PHY-0652356 (R.S.) is gratefully acknowledged. 
In order to state our main result let $1 \leq p<\infty$ and $0<s<1$ with $p s \neq 1$ and denote by $\mathcal{W}_{p}^{s}\left(\mathbb{R}_{+}^{N}\right)$ the completion of $C_{0}^{\infty}\left(\mathbb{R}_{+}^{N}\right)$ with respect to the left side of (1.1). It is a consequence of the Hardy inequality that this completion is a space of functions. Moreover, it is well-known that for $p s<1, \mathcal{W}_{p}^{s}\left(\mathbb{R}_{+}^{N}\right)$ coincides with the completion of $C_{0}^{\infty}\left(\overline{\mathbb{R}_{+}^{N}}\right)$.

Theorem 1.1 (Sharp fractional Hardy inequality). Let $N \geq 1,1 \leq p<\infty$ and $0<s<1$ with $p s \neq 1$. Then for all $u \in \mathcal{W}_{p}^{s}\left(\mathbb{R}_{+}^{N}\right)$,

$$
\iint_{\mathbb{R}_{+}^{N} \times \mathbb{R}_{+}^{N}} \frac{|u(x)-u(y)|^{p}}{|x-y|^{N+p s}} d x d y \geq \mathcal{D}_{N, p, s} \int_{\mathbb{R}_{+}^{N}} \frac{|u(x)|^{p}}{x_{N}^{p s}} d x
$$

with

$$
\mathcal{D}_{N, p, s}:=2 \pi^{(N-1) / 2} \frac{\Gamma((1+p s) / 2)}{\Gamma((N+p s) / 2)} \int_{0}^{1}\left|1-r^{(p s-1) / p}\right|^{p} \frac{d r}{(1-r)^{1+p s}} .
$$

The constant $\mathcal{D}_{N, p, s}$ is optimal. If $p=1$ and $N=1$, equality holds iff $u$ is proportional to a non-increasing function. If $p>1$ or if $p=1$ and $N \geq 2$, the inequality is strict for any function $0 \not \equiv u \in \mathcal{W}_{p}^{s}\left(\mathbb{R}_{+}^{N}\right)$.

For $p \geq 2$, inequality (1.3) holds even with a remainder term.

Theorem 1.2 (Sharp Hardy inequality with remainder). Let $N \geq 1,2 \leq p<\infty$ and $0<s<1$ with $p s \neq 1$. Then for all $u \in \mathcal{W}_{p}^{s}\left(\mathbb{R}_{+}^{N}\right)$ and $v:=x_{N}^{(1-p s) / p} u$,

$$
\begin{aligned}
\iint_{\mathbb{R}_{+}^{N} \times \mathbb{R}_{+}^{N}} \frac{|u(x)-u(y)|^{p}}{|x-y|^{N+p s}} d x d y & -\mathcal{D}_{N, p, s} \int_{\mathbb{R}_{+}^{N}} \frac{|u(x)|^{p}}{x_{N}^{p s}} d x \\
& \geq c_{p} \iint_{\mathbb{R}_{+}^{N} \times \mathbb{R}_{+}^{N}} \frac{|v(x)-v(y)|^{p}}{|x-y|^{N+p s}} \frac{d x}{x_{N}^{(1-p s) / 2}} \frac{d y}{y_{N}^{(1-p s) / 2}}
\end{aligned}
$$

where $\mathcal{D}_{N, p, s}$ is given by (1.4) and $0<c_{p} \leq 1$ is given by

$$
c_{p}:=\min _{0<\tau<1 / 2}\left((1-\tau)^{p}-\tau^{p}+p \tau^{p-1}\right) .
$$

If $p=2$, then (1.5) is an equality with $c_{2}=1$.

We conclude this section by mentioning an open problem concerning fractional Hardy-Sobolev-Maz'ya inequalities. If $p \geq 2$ and $0<s<1$ with $1<p s<N$, is it true that the left side of (1.5) is bounded from below by a positive constant times

$$
\left(\int_{\mathbb{R}_{+}^{N}}|u|^{q} d x\right)^{p / q}, \quad q=N p /(N-p s) ?
$$

The analogous estimate for $s=1$,

$$
\int_{\mathbb{R}_{+}^{N}}|\nabla u|^{p} d x-\left(\frac{p-1}{p}\right)^{p} \int_{\mathbb{R}_{+}^{N}} \frac{|u(x)|^{p}}{x_{N}^{p}} d x \geq \sigma_{N, p}\left(\int_{\mathbb{R}_{+}^{N}}|u|^{q} d x\right)^{p / q}, \quad q=N p /(N-p),
$$


is due to Maz'ya (for $p=2$ ) [M] and Barbatis-Filippas-Tertikas (for $2<p<N$ ) $\left[\mathrm{BFT}\right.$; see also [BFL] for the sharp value of $\sigma_{3,2}$. The proof of (1.7) is based on the analogue of (1.5),

$$
\int_{\mathbb{R}_{+}^{N}}|\nabla u|^{p} d x-\left(\frac{p-1}{p}\right)^{p} \int_{\mathbb{R}_{+}^{N}} \frac{|u(x)|^{p}}{x_{N}^{p}} d x \geq c_{p} \int_{\mathbb{R}_{+}^{N}}|\nabla v|^{p} x_{N}^{p-1} d x, \quad u=x_{N}^{(p-1) / p} v .
$$

\section{Proofs}

2.1. General Hardy inequalities. This subsection is a quick reminder of the results in [FS]. Throughout we fix $N \geq 1, p \geq 1$ and an open set $\Omega \subset \mathbb{R}^{N}$. Let $k$ be a nonnegative measurable function on $\Omega \times \Omega$ satisfying $k(x, y)=k(y, x)$ for all $x, y \in \Omega$ and define

$$
E[u]:=\iint_{\Omega \times \Omega}|u(x)-u(y)|^{p} k(x, y) d x d y .
$$

Our key assumption for proving a Hardy inequality for the functional $E$ is the following.

Assumption 2.1. Let $\omega$ be an a.e. positive, measurable function on $\Omega$. There exists a family of measurable functions $k_{\varepsilon}, \varepsilon>0$, on $\Omega \times \Omega$ satisfying $k_{\varepsilon}(x, y)=k_{\varepsilon}(y, x)$, $0 \leq k_{\varepsilon}(x, y) \leq k(x, y)$ and

$$
\lim _{\varepsilon \rightarrow 0} k_{\varepsilon}(x, y)=k(x, y)
$$

for a.e. $x, y \in \Omega$. Moreover, the integrals

$$
V_{\varepsilon}(x):=2 \omega(x)^{-p+1} \int_{\Omega}(\omega(x)-\omega(y))|\omega(x)-\omega(y)|^{p-2} k_{\varepsilon}(x, y) d y
$$

are absolutely convergent for a.e. $x$, belong to $L_{1, \text { loc }}(\Omega)$ and $V:=\lim _{\varepsilon \rightarrow 0} V_{\varepsilon}$ exists weakly in $L_{1, \text { loc }}(\Omega)$, i.e., $\int V_{\varepsilon} g d x \rightarrow \int V g d x$ for any bounded $g$ with compact support in $\Omega$.

The following abstract Hardy inequality was proved in [FS] in the special case $\Omega=\mathbb{R}^{N}$. The general case considered here is proved by exactly the same arguments.

Proposition 2.2. Under Assumption 2.1, for any $u$ with compact support in $\Omega$ and $E[u]$ and $\int V_{+}|u|^{p} d x$ finite one has

$$
E[u] \geq \int_{\Omega} V(x)|u(x)|^{p} d x .
$$

For $p \geq 2$, a stronger version of (2.3) is valid which includes a remainder term.

Proposition 2.3. Let $p \geq 2$. Under Assumption 2.1, for any $u$ with compact support in $\Omega$ write $u=\omega v$ and assume that $E[u], \int V_{+}|u|^{p} d x$, and

$$
E_{\omega}[v]:=\iint_{\Omega \times \Omega}|v(x)-v(y)|^{p} \omega(x)^{\frac{p}{2}} k(x, y) \omega(x)^{\frac{p}{2}} d x d y
$$


are finite. Then

$$
E[u]-\int_{\Omega} V(x)|u(x)|^{p} d x \geq c_{p} E_{\omega}[v]
$$

with $c_{p}$ from (1.6). If $p=2$, then (2.4) is an equality with $c_{2}=1$.

2.2. Proof of Theorem 1.1. Throughout this subsection we fix $N \geq 1,0<s<1$ and $p \neq 1 / s$ and we abbreviate

$$
\alpha:=(1-p s) / p .
$$

We will deduce the sharp Hardy inequality (1.3) using the general approach in the previous subsection with the choice

$$
\omega(x)=x_{N}^{-\alpha}, \quad k(x, y)=|x-y|^{-N-p s}, \quad V(x)=\mathcal{D}_{N, p, s} x_{N}^{-p s} .
$$

The key observation is

Lemma 2.4. One has uniformly for $x$ from compacts in $\mathbb{R}_{+}^{N}$

$2 \lim _{\varepsilon \rightarrow 0} \int_{y \in \mathbb{R}_{+}^{N},\left|x_{N}-y_{N}\right|>\varepsilon}\left(\omega\left(x_{N}\right)-\omega\left(y_{N}\right)\right)\left|\omega\left(x_{N}\right)-\omega\left(y_{N}\right)\right|^{p-2} k(x, y) d y=\frac{\mathcal{D}_{N, p, s}}{x_{N}^{p s}} \omega(x)^{p-1}$

with $\mathcal{D}_{N, p, s}$ from (1.4).

Proof. First, let $N=1$. Then it follows from [FS, Lem. 3.1] that

$$
2 \lim _{\varepsilon \rightarrow 0} \int_{y>0,|x-y|>\varepsilon}(\omega(x)-\omega(y))|\omega(x)-\omega(y)|^{p-2} k(x, y) d y=\frac{\mathcal{D}_{1, p, s}}{x^{p s}} \omega(x)^{p-1}
$$

uniformly for $x$ from compacts in $(0, \infty)$. To be more precise, in [FS, Lem. 3.1] the $y$-integral was extended over the whole axis. Therefore the difference between the constant $\mathcal{C}_{1, s, p}$ in $[\mathrm{FS},(3.2)]$ and our $\mathcal{D}_{1, p, s}$ here comes from the absolutely convergent integral

$$
2 \int_{-\infty}^{0}(\omega(x)-\omega(|y|))|\omega(x)-\omega(|y|)|^{p-2} \frac{d y}{(x-y)^{1+p s}}
$$

This proves the assertion for $N=1$. In order to extend the assertion to higher dimensions we use the fact (see $[\mathrm{AS},(6.2 .1)]$ ) that

$$
\begin{aligned}
\int_{\mathbb{R}^{N-1}} \frac{d y^{\prime}}{\left(\left|x^{\prime}-y^{\prime}\right|^{2}+m^{2}\right)^{(N+p s) / 2}} & =\left|\mathbb{S}^{N-2}\right| m^{-1-p s} \int_{0}^{\infty} \frac{r^{N-2} d r}{\left(r^{2}+1\right)^{(N+p s) / 2}} \\
& =\frac{1}{2}\left|\mathbb{S}^{N-2}\right| m^{-1-p s} \frac{\Gamma((N-1) / 2) \Gamma((1+p s) / 2)}{\Gamma((N+p s) / 2)}
\end{aligned}
$$

for $N \geq 2$. Recalling $\left|\mathbb{S}^{N-2}\right|=2 \pi^{(N-1) / 2} / \Gamma((N-1) / 2)$ concludes the proof.

Proof of Theorem 1.1. According to Lemma 2.4, Assumption 2.1] is satisfied with kernel $k_{\varepsilon}(x, y)=|x-y|^{-N-p s} \chi_{\left\{\left|x_{N}-y_{N}\right|>\varepsilon\right\}}$. Hence inequality (1.3) for $u \in C_{0}^{\infty}\left(\mathbb{R}_{+}^{N}\right)$ follows from Proposition 2.2. By density it holds for all $u \in \mathcal{W}_{p}^{s}\left(\mathbb{R}_{+}^{N}\right)$. Strictness for $p>1$ follows by the same argument as in [FS]. In order to discuss equality in (1.3) for $p=1$ we first note that for equality it is necessary that $u$ is proportional to a non-negative 
function, which we assume henceforth. From [FS, (2.18)] we see that equality holds iff for a.e. $x$ and $y$ with $\omega\left(x_{N}\right)>\omega\left(y_{N}\right)$ (that is, $\left.x_{N}<y_{N}\right)$ one has

$$
\left|\omega\left(x_{N}\right) v(x)-\omega\left(y_{N}\right) v(y)\right|-\left(\omega\left(x_{N}\right) v(x)-\omega\left(y_{N}\right) v(y)\right)=0
$$

for $v(x):=\omega\left(x_{N}\right)^{-1} u(x)$. Since for numbers $a, b \geq 0$ the equality $|a-b|-(a-b)=0$ holds iff $b \leq a$, we conclude that for a.e. $x$ and $y$ with $x_{N}<y_{N}$ one has $\omega\left(y_{N}\right) v(y) \leq$ $\omega\left(x_{N}\right) v(x)$, that is $u(y) \leq u(x)$. If $N=1$ this means that $u$ is non-increasing. If $N \geq 2$ one sees that for a function $u$ with this property the integral $\int_{\mathbb{R}_{+}^{N}}|u| x_{N}^{-s} d x$ is infinite, unless $u \equiv 0$. This proves the strictness assertion in Theorem 1.1.

The fact that the constant is sharp for $N=1$ was shown in [FS] (with $\mathbb{R}_{+}$replaced by $\mathbb{R}$, but this only leads to trivial modifications). In order to prove sharpness in higher dimensions we consider functions of the form $u_{n}(x)=\chi_{n}\left(x^{\prime}\right) \varphi\left(x_{N}\right)$, where

$$
\chi_{n}\left(x^{\prime}\right)= \begin{cases}1 & \text { if }\left|x^{\prime}\right| \leq n, \\ n+1-\left|x^{\prime}\right| & \text { if } n<\left|x^{\prime}\right|<n+1, \\ 0 & \text { if }\left|x^{\prime}\right| \geq n+1 .\end{cases}
$$

An easy calculation using (2.7) shows that

$$
\frac{\iint_{\mathbb{R}_{+}^{N} \times \mathbb{R}_{+}^{N}} \frac{\left|u_{n}(x)-u_{n}(y)\right|^{p}}{|x-y|^{N+p s}} d x d y}{\int_{\mathbb{R}_{+}^{N}} \frac{\left|u_{n}(x)\right|^{p}}{x_{N}^{p s}} d x} \rightarrow A \frac{\iint_{\mathbb{R}_{+} \times \mathbb{R}_{+}} \frac{\left|\varphi\left(x_{N}\right)-\varphi\left(y_{N}\right)\right|^{p}}{\left|x_{N}-y_{N}\right|^{1+p s}} d x_{N} d y_{N}}{\int_{\mathbb{R}_{+}} \frac{|\varphi(x)|^{p}}{x_{N}^{p s}} d x_{N}}
$$

as $n \rightarrow \infty$ with $A:=\frac{1}{2}\left|\mathbb{S}^{N-2}\right| \Gamma((N-1) / 2) \Gamma((1+p s) / 2) / \Gamma((N+p s) / 2)$. Since $A=\mathcal{D}_{N, p, s} / \mathcal{D}_{1, p, s}$, sharpness of $\mathcal{D}_{N, p, s}$ for $N \geq 2$ follows from sharpness of $\mathcal{D}_{1, p, s}$ for $N=1$.

Proof of Theorem 1.2. Inequality (1.2) follows immediately from Proposition 2.3.

\section{REFERENCES}

[AS] M. Abramowitz, I. A. Stegun, Handbook of mathematical functions with formulas, graphs, and mathematical tables. Reprint of the 1972 edition. Dover Publications, New York, 1992.

[BFT] G. Barbatis, S. Filippas, A. Tertikas, A unified approach to improved L ${ }^{p}$ Hardy inequalities with best constants. Trans. Amer. Math. Soc. 356 (2004), no. 6, 2169-2196.

[BFL] R. D. Benguria, R. L. Frank, M. Loss, The sharp constant in the Hardy-Sobolev-Maz'ya inequality in the three dimensional upper half-space. Math. Res. Lett. 15 (2008), no. 4, 613622 .

[BD] K. Bogdan, B. Dyda, The best constant in a fractional Hardy inequality, Preprint (2008), arXiv:0807.1825v1.

[BBM] J. Bourgain, H. Brezis, P. Mironescu, Limiting embedding theorems for $W^{s, p}$ when s 11 and applications. J. Anal. Math. 87 (2002), 77-101.

[D] B. Dyda, A fractional order Hardy inequality. Illinois J. Math. 48 (2004), no. 2, 575-588.

[FS] R. L. Frank, R. Seiringer, Non-linear ground state representations and sharp Hardy inequalities. J. Funct. Anal. 255 (2008), 3407-3430.

[KMP] N. Krugljak, L. Maligranda, L. E. Persson, On an elementary approach to the fractional Hardy inequality. Proc. Amer. Math. Soc. 128 (2000), no. 3, 727-734.

[M] V. G. Maz'ya, Sobolev spaces. Springer, 1985. 
[MS] V. G. Maz'ya, T. Shaposhnikova, On the Bourgain, Brezis, and Mironescu theorem concerning limiting embeddings of fractional Sobolev spaces. J. Funct. Anal. 195 (2002), no. 2, 230-238. Erratum: J. Funct. Anal. 201 (2003), no. 1, 298-300.

Rupert L. Frank, Department of Mathematics, Princeton University, Washington RoAd, Princeton, NJ 08544, USA

E-mail address: rlfrank@math.princeton.edu

Robert Seiringer, Department of Physics, Princeton University, P. O. Box 708, Princeton, NJ 08544, USA

E-mail address: rseiring@princeton.edu 